CONTROL ID: 2202234

TITLE: A numerical design of a frequency-based analytical model for demagnetization detection in axial flux permanent magnet synchronous machines AUTHORS (LAST NAME, FIRST NAME): De Bisschop, Jan ${ }^{1}$; Abdallh, Ahmed ${ }^{2}$; Sergeant, Peter ${ }^{1,2}{ }^{2}$; Dupre , Luc $^{2}$
INSTITUTIONS (ALL):

1. Department of Industrial Technology and Construction, Ghent University, Ghent, Belgium.

2. Department of Electrical Energy, Systems and Automation, Ghent University, Ghent, Belgium.

\title{
ABSTRACT BODY:
}

Digest Body: Introduction Axial flux permanent magnet synchronous machines (AFPMSMs) have been extensively used in different applications due to their excellent performances [1]. Particularly, AFPMSMs are well dedicated for applications that acquire high power density, such as substantial energy applications. In practice, these types of machines suffer from different faults, such as a rotor eccentricity and permanent magnet (PM) demagnetization, which decrease their reliability. Early detection of these faults can lead to mitigation of catastrophic failures. Recently, an inverse problem based methodology was presented to detect the demagnetization faults in a yokeless AFPMSM [2]. This inverse problem interprets the measured voltage waveforms into a frequency-based analytical model that takes the PM demagnetization into consideration. This analytical model solves Maxwell's equations for a predefined number of time and space harmonics. Modeling few harmonic orders lead to inaccurate representations of the machine electromagnetic behavior, and consequently, inaccurate identification results. On contrary, large number of harmonic representation dramatically increases the computational time especially in iterative inverse problems. Hence, there is a definitely need to optimize the number of the used time and space harmonics. In this paper, we aim at determining the optimal values of the time and space harmonics used in the frequency-based analytical model, for a better detection of PM demagnetization fault in the AFPMSM. To this end, a numerical design of the analytical model is proposed based on a stochastic Cramér-Rao lower bound technique. Moreover, the analytical model presented in [2] has some drawbacks, such as not perfectly simulating the asymmetrical defects. These drawbacks have been overcome in the present model. A frequency-based analytical model The model analytically solves the vector potential differential equation in five regions (see Figure 1). The regions are respectively the PMs of the right rotor, the air gap of the right rotor, the stator, the air gap of the left rotor and the PMs of the left rotor. The solution assumes a slotless machine and soft magnetic materials with infinite permeability. The stator is resized so that the tangential reluctance equals those of the real stator. This accounts for the stator slots in the third region. In the air gap regions, the stator slots are introduced by means of a permeance function. The main advantage of the five-region model, over the threeregion one presented in [2], is the ability to account for asymmetrical faults. The magnetization in the first and fifth region is implemented as a series of square waveforms over the circumference, with every waveform represents a PM. In the healthy state, all these waveforms have the same magnetization, corresponding to the PM remanence. The demagnetization defect is modeled by means of a magnetization array. This array consists of values ranging from 0 to 1 , called the magnetization coefficients; 0 means completely demagnetized and 1 means a healthy state of a PM. These values are multiplied with the square wave. In this way, the squares of a demagnetized magnet are lowered relative to their magnetization coefficient. The flux density field of the PMs taking into account the armature reaction gives the total air gap flux density field. Based on this total field, the back-EMF for each stator coil is calculated. From this phase back-EMF and the terminal voltages are computed. The model is built in the frequency domain, in which a predefined finite time and space harmonic orders are utilized. Optimal number of the used harmonic order need to be known. A numerical design algorithm The stochastic Cramér-Rao lower bound technique (sCRLB) is a well-known approach to estimate the lower bound error in the identified parameters of inverse problems [3]. Generally speaking, SCRLB computes the Fisher Information Matrix (FIM), which is based on the sensitivity analysis of the forward model to the unknown parameters, i.e. the PM demagnetization factor in our case study. According to the theory of sCRB, the error lower bounds of the identified parameters are defined as the inverse of the FIM determinant [4]. In this paper, we use the SCRLB to numerically design the forward model of an AFPMSM for a better and fast detection of PM demagnetization faults, i.e. select a priori the optimal values of the time and space harmonics. To accomplish this target, we solve a discrete optimization problem which maximizes the determinant of the FIM, i.e. minimizes the possible identification error. In other words, the optimization problem selects the most sensitive time and space harmonic orders that at least need to be modeled in order to obtain the best identification results. Results Figure 2 shows the per unit values of the FIM determinant for different time harmonic orders in healthy and faulty conditions. It 
is clear that the 6th time harmonic order is essential to reasonably simulate this specific AFPMSM. The effect of the number of space harmonics is found to be negligible. In the full paper, the detailed description of the coupled stochastic-optimization algorithm is given. Moreover, the effects of different fault conditions, machine topologies, as well as, the uncertainties in some machine model parameters are discussed and analyzed.

References: [1] F. G. Capponi, G. De Donato, and F. Caricchi, "Recent advances in axial-flux permanent-magnet machine technology," IEEE Trans. Ind. Appl., vol. 48, no. 6, pp. 2190-2205, Nov./Dec. 2012. [2] J. De Bisschop, A. Abdallh, P. Sergeant, and L. Dupré, "Identification of demagnetization faults in axial flux permanent magnet synchronous machines using an inverse problem coupled with an analytical model," IEEE Trans. Magn., vol. 50, no. 11, Art. No. 8104804, November 2014. [3] A. Abdallh , G. Crevecoeur, and L. Dupré, "Optimal needles placement for the accurate magnetic material quantification based on uncertainty analysis in the inverse approach," Measurement Science and Technology, vol. 21, no. 11, 115703 (16pp), 2010. [4] A. Emery, A. Nenarokomov, and T. Fadale, "Uncertainties in parameter estimation: the optimal experiment," International Journal of Heat Mass Transfer, vol. 43, pp. 3331-3339, 2000.

KEYWORDS: Inverse problems, Demagnetization detection , Numerical design. 


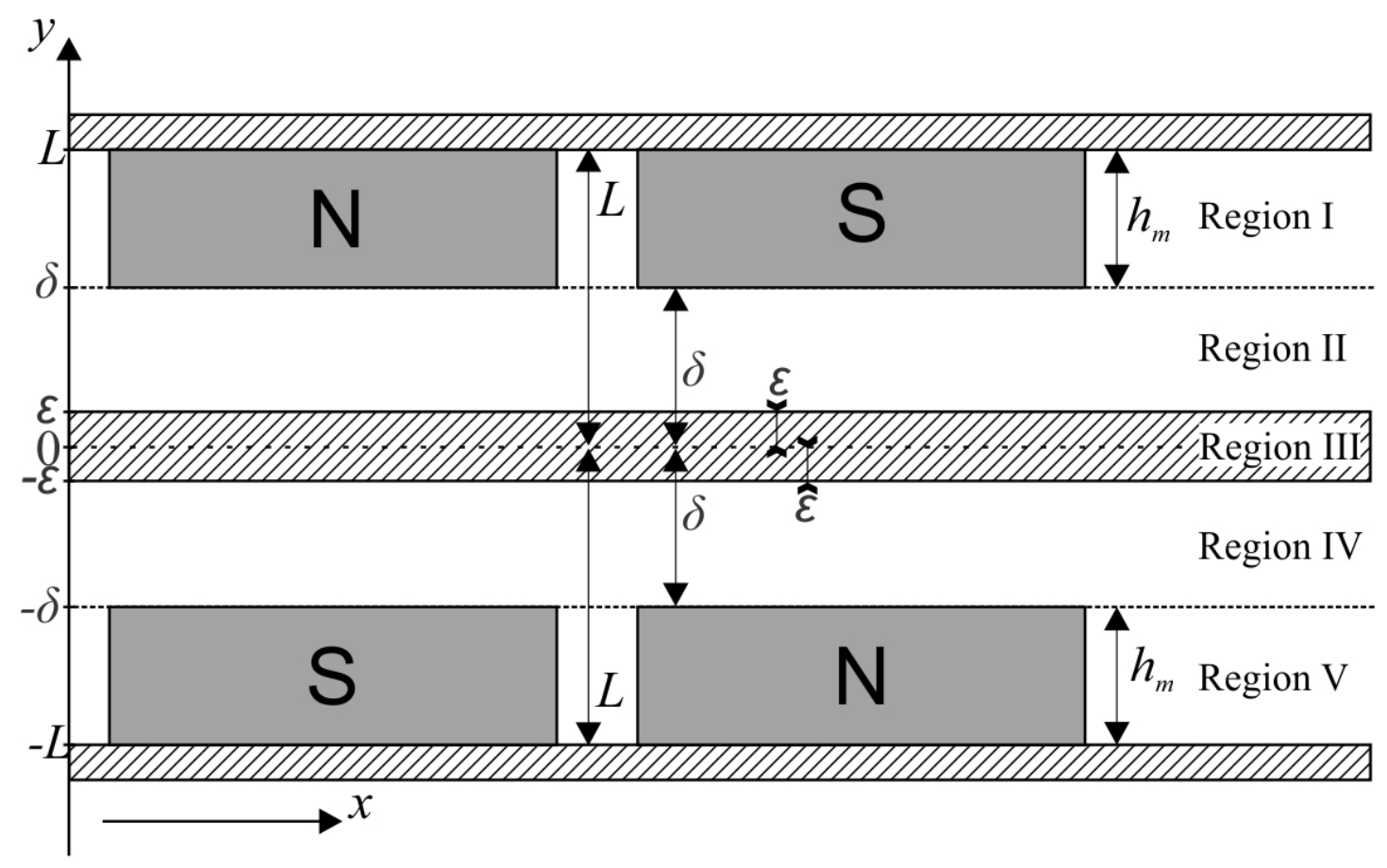

Figure 1: Overview of the 5 regions used in the analytical model. 

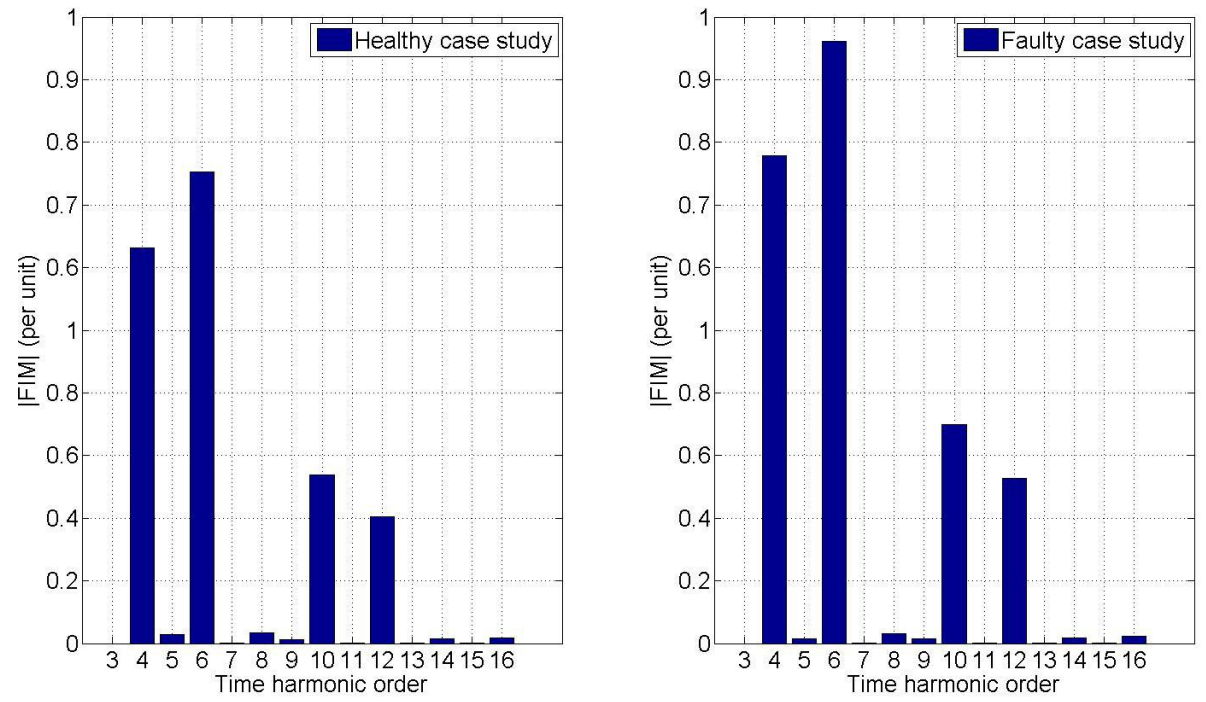

Figure 2: FIM determinant per unit values for different time harmonic orders in healthy and faulty conditions. 
CONTACT (NAME ONLY): Jan De Bisschop

CONTACT (E-MAIL ONLY): Jan.DeBisschop@UGent.be

PRESENTATION TYPE: Poster

CURRENT CATEGORY: 4. Electric machines, power devices \& electromagnetics

CURRENT SUB-CATEGORY: e. Electromagnetic modeling and analysis in electric machines and power grid 